\title{
FUNDAMENTAL SOLUTIONS TO KOLMOGOROV EQUATIONS VIA REDUCTION TO CANONICAL FORM
}

\author{
JOANNA GOARD
}

Received 25 January 2006; Revised 11 May 2006; Accepted 13 June 2006

This paper finds fundamental solutions to the backward Kolmogorov equations, usually interpretable as transition density functions for variables $x$ that follow certain stochastic processes of the form $d x=A(x, t) d t+c x^{\gamma} d X$ and $d x=A(x, t) d t+\sqrt{\alpha_{1}+\alpha_{2} x+\alpha_{3} x^{2}} d X$. This is achieved by first reducing the relevant PDEs that the density functions satisfy to their canonical form. These stochastic processes have direct realistic applications in the modeling of financial assets.

Copyright (c) 2006 Joanna Goard. This is an open access article distributed under the Creative Commons Attribution License, which permits unrestricted use, distribution, and reproduction in any medium, provided the original work is properly cited.

\section{Introduction}

Any endeavor to calculate the theoretical price of certain financial derivative products, such as options and bonds, begins with the establishment of a dynamic process followed by the underlying asset. It is well known that under the assumption that an underlying stock with price $S$ follows the lognormal process

$$
d S=\mu S d t+\sigma S d X
$$

Black and Scholes [4] derived their celebrated partial differential equation (PDE) for the valuation of European options. Note that in (1.1) and throughout this paper, $d X$ denotes an increment in a Wiener process so that $d X \sim N(0, \sqrt{d t})$.

Similarly when the short-term interest rate $r$ follows the process

$$
d r=u(r, t) d t+w(r, t) d X,
$$

the price of a zero-coupon bond $V(r, t)$ with expiry at time $t=T$ will satisfy the PDE

$$
\frac{\partial V}{\partial t}+\frac{w(r, t)^{2}}{2} \frac{\partial^{2} V}{\partial r^{2}}+(u(r, t)-\lambda(r, t) w(r, t)) \frac{\partial V}{\partial r}-r V=0
$$

subject to $V(r, T)=1$, and where $\lambda(r, t)$ is the market price of risk (see, e.g., [16]). 
2 Fundamental solutions to Kolmogorov equations

In order to understand the probabilistic properties of random walks such as (1.1) and (1.2) which we write in general as

$$
d x=A(x, t) d t+B(x, t) d X
$$

it is necessary to find transition density functions (TDFs), $p\left(x, t ; y, t^{\prime}\right)$, of the underlying process. This density function $p\left(x, t ; y, t^{\prime}\right)$ is defined by

$$
\operatorname{Pr}\left(a<x<b \text { at time } t^{\prime} \mid x \text { at time } t\right)=\int_{a}^{b} p\left(x, t ; y, t^{\prime}\right) d y .
$$

The variables $x$ and $t$ can be thought of as the current variables and $y$ and $t^{\prime}$ as the future variables. The TDF satisfies the backward Kolmogorov equation

$$
\frac{\partial p}{\partial t}+\frac{1}{2} B(x, t)^{2} \frac{\partial^{2} p}{\partial x^{2}}+A(x, t) \frac{\partial p}{\partial x}=0
$$

involving the derivatives with respect to the current state and time, subject to

$$
p\left(x, t^{\prime} ; y, t^{\prime}\right)=\delta(x-y) \text {. }
$$

Also, at each point in time for $x, y \geq 0$, TDFs satisfy the condition

$$
\int_{0}^{\infty} p\left(x, t ; y, t^{\prime}\right) d y=1
$$

When (1.4) describes a risk-neutral process, TDFs can be used to value financial derivatives such as European call (and put) options, giving the holder of the options the right to buy (sell) the asset with value $x$, at some future time $T$ for a price $K$ agreed upon at the current time $t$. The price for the call option contract is then

$$
\begin{aligned}
C(x, t ; K, T) & =e^{-r(T-t)} E(\max (x-K, 0)) \\
& =e^{-r(T-t)} \int_{K}^{\infty}(y-K) p(x, t ; y, T) d y,
\end{aligned}
$$

where $r$ is the risk-free interest rate. Similarly, the price for the put option contract is

$$
\begin{aligned}
P(x, t ; K, T) & =e^{-r(T-t)} E(\max (K-x, 0)) \\
& =e^{-r(T-t)} \int_{0}^{K}(K-y) p(x, t ; y, T) d y .
\end{aligned}
$$

Given a fundamental solution, the solution to the Cauchy problem can be obtained from the given initial data by convolution. In [9], Craddock and Platen find some fundamental solutions when $B(x)=\sqrt{2 x}$ and $A(x)=f(x)$, that is, of

$$
\frac{\partial p}{\partial \tau}=x \frac{\partial^{2} p}{\partial x^{2}}+f(x) \frac{\partial p}{\partial x}
$$


where $\tau=t^{\prime}-t$. When (1.6c) is satisfied, this corresponds to finding TDFs of the process $x$, which follows

$$
d x=f(x) d t+\sqrt{2 x} d X
$$

The method they employed was to perform a classical symmetry analysis of (1.9), and then using certain symmetries found, write the corresponding form of the transformed solutions $p^{*}$ in terms of another arbitrary solution $p$, that is,

$$
p^{*}(x, t)=p(x, t) F(x, t ; \epsilon) .
$$

The symmetry transformations were chosen so as to achieve an integral transform, preferably if possible, the Laplace transform of the fundamental solution. Thus, if possible, the symmetries were chosen so that $F(x, 0 ; \epsilon)=e^{-\lambda x}$, where $\lambda$ is some function of $\epsilon$. Then by choosing the solution $p=1$ of (1.9), the fundamental solution of (1.9) is the Laplace inverse of $\tilde{p}$, the new solution with initial condition $e^{-\lambda x}$, as for $x, y \geq 0$,

$$
\tilde{p}(x, \tau)=\int_{0}^{\infty} e^{-\lambda y} p\left(x, \tau ; y, t^{\prime}\right) d y .
$$

$\tilde{p}$ was called the characteristic solution (the Laplace transform of the fundamental solution).

Craddock and Platen found fundamental solutions of (1.9) when $f$ satisfied the Riccati equations

$$
\begin{gathered}
x f^{\prime}-f+\frac{1}{2} f^{2}=A_{1} x+B_{1}, \\
x f^{\prime}-f+\frac{1}{2} f^{2}=A_{1} x^{2}+B_{1} x+C_{1}, \\
x f^{\prime}-f+\frac{1}{2} f^{2}=A_{1} x^{3 / 2}+B_{1} x^{2}+C_{1} x-\frac{3}{8} .
\end{gathered}
$$

For functions $f$ that satisfied (1.13a), the characteristic solution was found in general. When $f$ satisfied relation (1.13b), solutions were proposed that may lead to characteristic solutions and when $f$ satisfied $(1.13 \mathrm{c})$ and $B_{1}=0$, the characteristic solution was given, but not its corresponding Laplace inverse.

It is known (see, e.g., [15]) that an evolution equation of the type

$$
P(x, t) u_{t}+Q(x, t) u_{x}+R(x, t) u_{x x}+S(x, t) u=0
$$

with $P, R \neq 0$, can be reduced via a suitable transformation to

$$
v_{\bar{t}}=v_{\overline{x x}}+Z(\bar{t}, \bar{x}) v .
$$

When the PDE (1.14) admits one additional symmetry operator (namely $\partial / \partial t$ ) to $\partial / \partial u$ and $\varphi(x, t)(\partial / \partial u)$, where $\varphi$ is any solution of (1.14), then (1.14) is reducible to

$$
v_{\bar{t}}=v_{\bar{x} \bar{x}}+Z(\bar{x}) v .
$$


If the PDE (1.14) has at least three more symmetries in addition to $u(\partial / \partial u)$ and $\varphi(x, t)(\partial / \partial u)$, where $\varphi$ is any solution of $(1.14)$, then (1.14) is reducible to

$$
v_{\bar{t}}=v_{\bar{x} \bar{x}}+\frac{\alpha}{\bar{x}^{2}} v
$$

where $\alpha$ is constant. If it has at least five other symmetries (to $u(\partial / \partial u)$ and $\varphi(x, t)(\partial / \partial u)$ ), then it is reducible to

$$
v_{\bar{t}}=v_{\overline{x x}}
$$

This system of classification affords a systematic approach to finding reductions. As (1.9) in the cases where $f$ satisfies $(1.13 \mathrm{a}),(1.13 \mathrm{~b})$, or $(1.13 \mathrm{c})$ has at least three extra symmetries beyond $p(\partial / \partial p)$ and $\varphi(x, \tau)(\partial / \partial p)$, where $\varphi(x, \tau)$ satisfies $(1.9),(1.9)$ is reducible to either (1.17) or (1.18). It is then possible to easily find the fundamental solutions in these cases by using the fundamental solutions of (1.17) and (1.18). This also avoids the necessity of finding suitable symmetries that lead to tractable integral transform inverses.

In [2], Albanese and Kuznetsov show that a stationary process of the form (1.4), where $A=A(x)$ and $B=B(x)$, can be transformed via a suitable change of variables and change of measure to a process that is driftless. This in fact corresponds to the reduction of (1.14) to canonical form. They then classify driftless processes that are solvable in the sense that the corresponding eigenfunction equation can be reduced by a change of measure and change of variables to a hypergeometric or confluent hypergeometric equation. This then could lead to TDFs as infinite series of hypergeometric (Gaussian or confluent) functions.

The driftless processes $d x=\left(a+b x+c x^{2}\right) d X$ (quadratic volatility) and $d x=x^{\gamma} d X$ (CEV driftless) have been well researched, and in [1] Albanese et al. show that these processes belong to the equivalence class of the family $d x=\left(\lambda_{0}+\lambda_{1} x\right) d t+\nu_{0} x^{\beta} d X$, where $\beta=0,1 / 2$. In turn, this family of processes is contained within the broader class that we consider in this paper.

The aim of this paper is to look for solutions of (1.6a)-(1.6b) for general functions $A$ and when $B(x, t)=c x^{\gamma}$ (Section 3) and $B(x, t)=\sqrt{\alpha_{1}+\alpha_{2} x+\alpha_{3} x^{2}}$ (Section 4), by first reducing (1.6a) to its canonical form. Rather than finding solutions to (1.6a) in terms of series of transcendental functions (such as in [2]), the focus here is on being able to solve the Cauchy problem by a single integration. When (1.6c) is satisfied (as in all our examples), the fundamental solutions can be viewed as the TDFs for the variable $x$ that follows the process (1.4). As economic conditions change with time, it is reasonable to expect that the instantaneous expected return of a given state variable, such as stock prices and bond yields, depends on time and price level. In fact, Fan et al. [10] and Goard and Hansen [13], among others, have shown how models with time-dependent parameters outperform those with a long-term reversion to a fixed mean. As such, we focus here on time-dependent, as well as time-independent drifts $A$. The processes $(1.4)$ with $B(x, t)=$ $c x^{\gamma}, 0 \leq \gamma<1$, are referred to as "constant elasticity of variance" processes and are often used to model stock prices. The limiting case $\gamma=1$ corresponds to the lognormal process (1.1) while the case $\gamma=1 / 2$ corresponds to the square-root process used by Cox et al. [7], and considered by Craddock and Platen [9]. When $\gamma<1$, the variance of the percentage return from the stock decreases with the stock price. From empirical studies, Beckers [3] 
suggests that an option pricing formula based on constant elasticity of variance diffusion could fit the market prices better than the Black-Scholes model.

In interest-rate modeling, Chan et al. [6] performed a comprehensive empirical analysis on one-factor interest-rate models of the form

$$
d r=(\alpha+\beta r) d t+\sigma r^{\gamma} d X
$$

They found that the most successful models in capturing the dynamics of the short rate were those that allowed the volatility of interest rate changes to be highly sensitive to the level of interest rate, in particular with $\gamma \geq 1$. Their unconstrained estimate of $\gamma$ was 1.499 .

In considering a diffusion term of the form $B(x, t)=\sqrt{\alpha_{1}+\alpha_{2} x+\alpha_{3} x^{2}}$, the three arbitrary constants allow for extra flexibility for parameter estimation. It includes the diffusion terms of Cox et al. [7] $\left(\alpha_{1}=\alpha_{3}=0\right)$, Ho and Lee [14] $\left(\alpha_{2}=\alpha_{3}=0\right)$, and the lognormal process (1.1) $\left(\alpha_{1}=\alpha_{2}=0\right)$.

A summary of the results is presented in Section 5.

\section{Reduction to canonical form}

Equations of the form (1.6a) with $B=B(x)$ can be reduced to the canonical form

$$
z_{x_{2}}=z_{x_{1} x_{1}}+Q\left(x_{1}, x_{2}\right) z
$$

via a transformation of the type

$$
\begin{aligned}
& x_{1}=x_{1}(x, t), \\
& x_{2}=x_{2}(x, t), \\
& z=H(x, t) p
\end{aligned}
$$

(see, e.g., Bluman [5]). Substituting (2.2) into (2.1), we find that the transformation

$$
\begin{gathered}
x_{1}=\int \frac{d x}{B(x)}, \\
x_{2}=\frac{t^{\prime}-t}{2}, \\
z=\alpha(x) e^{k(x) t} p,
\end{gathered}
$$

where

$$
\frac{\alpha^{\prime}(x)}{\alpha(x)}+k^{\prime}(x) t=\frac{A}{B^{2}}-\frac{B^{\prime}}{2 B}
$$

reduces $(1.6 \mathrm{a})$ to $(2.1)$ with

$$
Q=-A_{x}+\left(\frac{2 B^{\prime}}{B}\right) A-\frac{A^{2}}{B^{2}}-\frac{\left(B^{\prime}\right)^{2}}{4}+\frac{B B^{\prime \prime}}{2}-2 k(x)
$$




\section{Fundamental solutions to Kolmogorov equations}

We look at the cases where $Q$ corresponds to

$$
\begin{array}{ll}
\text { (I) } & \frac{k_{1}}{x_{1}^{2}}+k_{2}, \\
\text { (II) } & \frac{k_{1}}{x_{1}^{2}}+k_{2} x_{1}^{2}+k_{3}, \\
\text { (III) } & k_{1} x_{1}^{2}+k_{2} x_{1}+k_{3},
\end{array}
$$

where $k_{1}, k_{2}$, and $k_{3}$ are constants, as in cases (I) and (II), (1.6a) could be further reduced to the form in (1.17) and in case (III), (1.6a) could be reduced further to a constantcoefficient PDE.

3. $B(x)=c x^{\gamma}$

In this section, $\gamma$ is assumed to be in the interval $0 \leq \gamma<1$ (i.e., the constant elasticity of variance case). However similar calculations apply for $\gamma>1$ and any variations in the solutions are noted. When $\gamma=1$, then the change of variable for $x$ in $(3.1 \mathrm{a})$ is $x_{1}=(1 / c) \ln x$. As this substitution for $x$ will not provide many useful tractable solutions for $A$ other than $A=\mu x$, for which the TDF is already known, we disregard this case.

When $B(x)=c x^{\gamma}$, from (2.3a) and (2.3b),

$$
\begin{gathered}
x_{1}=\frac{1}{c(1-\gamma)} x^{1-\gamma}, \\
x_{2}=\frac{t^{\prime}-t}{2},
\end{gathered}
$$

and $z$ is as given from $(2.3 \mathrm{c})$ and (2.3d).

We consider separately the cases where $A=A(x)$ and $A=A(x, t)$.

3.1. $A=A(x)$. In this case, we can let $k(x)=0$ in $(2.3 \mathrm{~d})$ so that from $(2.3 \mathrm{c})$ and $(2.3 \mathrm{~d})$,

$$
z=x^{-\gamma / 2} e^{1 / c^{2} \int\left(A(x) / x^{2 \gamma}\right) d x} p
$$

and from (2.4),

$$
Q=-A^{\prime}+\frac{2 \gamma}{x} A-\frac{1}{c^{2} x^{2 \gamma}} A^{2}+\frac{c^{2} \gamma^{2}}{4} x^{2 \gamma-2}-\frac{c^{2} \gamma}{2} x^{2 \gamma-2}
$$

We now consider separately the cases where $Q$ reduces to the forms $(2.5 a)-(2.5 c)$.

3.1.1. $Q=\left(k_{1} / x_{1}^{2}\right)+k_{2}$. For the case corresponding to (2.5a), we require that $A$ satisfy

$$
A^{\prime}-\frac{2 \gamma}{x} A+\frac{1}{c^{2} x^{2 \gamma}} A^{2}=m x^{2 \gamma-2}+n
$$


where

$$
\begin{gathered}
m=-k_{1} c^{2}(1-\gamma)^{2}+\frac{c^{2} \gamma^{2}}{4}-\frac{c^{2} \gamma}{2}, \\
n=-k_{2} .
\end{gathered}
$$

Then (1.6a) reduces to

$$
z_{x_{2}}=z_{x_{1} x_{1}}+\left(\frac{k_{1}}{x_{1}^{2}}+k_{2}\right) z
$$

which needs to be solved subject to

$$
z\left(x_{1}, 0\right)=G\left(x_{1}\right) \delta\left(q x_{1}^{1 /(1-\gamma)}-y\right),
$$

where

$$
\begin{aligned}
G\left(x_{1}\right) & =\left[c(1-\gamma) x_{1}\right]^{-\gamma / 2(1-\gamma)} \exp \left[\frac{1}{c^{2}} \int^{q x_{1}^{1 /(1-\gamma)}} \frac{A(x)}{x^{2 \gamma}} d x\right], \\
q & =(c(1-\gamma))^{1 /(1-\gamma)} .
\end{aligned}
$$

The solution to (3.4a)-(3.4b) for $-3 / 4 \leq k_{1} \leq 1 / 4$ that satisfies $z=0$ at $x_{1}=0$ is

$$
z\left(x_{1}, x_{2}\right)=\frac{e^{k_{2} x_{2}}}{2 x_{2}} \int_{0}^{\infty} \sqrt{x_{1}} \sqrt{\xi} e^{-\left(\left(x_{1}^{2}+\xi^{2}\right) / 4 x_{2}\right)} I_{\nu}\left(\frac{\xi x_{1}}{2 x_{2}}\right) G(\xi) \delta\left(q \xi^{1 /(1-\gamma)}-y\right) d \xi
$$

where $v^{2}=1 / 4-k_{1}$ so that

$$
\begin{aligned}
z\left(x_{1}, x_{2}\right)= & \frac{\sqrt{x_{1}}}{2 x_{2}} e^{k_{2} x_{2}} \frac{y^{1 / 2-2 \gamma}}{c^{3 / 2}(1-\gamma)^{1 / 2}} \exp \left\{-\left(\frac{c^{2}(1-\gamma)^{2} x_{1}^{2}+y^{2-2 \gamma}}{4 c^{2}(1-\gamma)^{2} x_{2}}\right)\right\} \\
& \times I_{\nu}\left(\frac{y^{1-\gamma} x_{1}}{2 c(1-\gamma) x_{2}}\right) \exp \left(\frac{1}{c^{2}} \int^{y} \frac{A(x)}{x^{2 \gamma}} d x\right) .
\end{aligned}
$$

Note that when $k_{1}=0$, we can write

$$
z\left(x_{1}, x_{2}\right)=\frac{y^{-3 \gamma / 2} e^{k_{2} x_{2}}}{2 c \sqrt{\pi x_{2}}} \exp \left(\frac{-\left(x_{1} c(1-\gamma)-y^{1-\gamma}\right)^{2}}{4 x_{2} c^{2}(1-\gamma)^{2}}\right) \exp \left(\frac{1}{c^{2}} \int^{y} \frac{A(x)}{x^{2 \gamma}} d x\right) .
$$

Then from (3.1a)-(3.1c),

$$
p\left(x, t ; y, t^{\prime}\right)=x^{\gamma / 2} \exp \left[\frac{-1}{c^{2}} \int \frac{A(x)}{x^{2 \gamma}} d x\right] z\left(\frac{1}{c(1-\gamma)} x^{1-\gamma}, \frac{t^{\prime}-t}{2}\right) .
$$

In general for $\gamma \geq 0, \gamma \neq 1$, we can write

$$
\begin{aligned}
p\left(x, t ; y, t^{\prime}\right)= & \frac{x^{1 / 2} y^{1 / 2-2 \gamma}}{c^{2}|1-\gamma|\left(t^{\prime}-t\right)} \exp \left\{\frac{k_{2}}{2}\left(t^{\prime}-t\right)\right\} \exp \left\{-\frac{\left(x^{2-2 \gamma}+y^{2-2 \gamma}\right)}{c^{2}(1-\gamma)^{2}\left(t^{\prime}-t\right)}\right\} \\
& \times I_{\nu}\left(\frac{y^{1-\gamma} x^{1-\gamma}}{c^{2}(1-\gamma)^{2}\left(t^{\prime}-t\right)}\right) \exp \left\{\frac{1}{c^{2}} \int^{y} \frac{A(x)}{x^{2 \gamma}} d x-\frac{1}{c^{2}} \int^{x} \frac{A(x)}{x^{2 \gamma}} d x\right\} .
\end{aligned}
$$


8 Fundamental solutions to Kolmogorov equations

Example 3.1. With $n=0$, a solution to (3.3) is

$$
A=x^{(2 \gamma-1)}\left[\frac{c k}{2}\left(\frac{D x^{k / c}-1}{D x^{k / c}+1}\right)+\frac{c^{2}}{2}\right]
$$

where $m$ and $D$ are arbitrary and $k=\sqrt{4 m+c^{2}} \neq 0$.

The corresponding stochastic process (1.4) for $x$ is given by

$$
d x=c x^{2 \gamma-1}\left[\frac{k}{2}\left(\frac{D x^{k / c}-1}{1+D x^{k / c}}\right)+\frac{c}{2}\right] d t+c x^{\gamma} d X
$$

with the associated TDF when $D=0$ given by

$$
p\left(x, t ; y, t^{\prime}\right)=\frac{x^{k / 2 c} y^{1-2 \gamma-k / 2 c}}{c^{2}|1-\gamma|\left(t^{\prime}-t\right)} \exp \left\{-\left(\frac{x^{2-2 \gamma}+y^{2-2 \gamma}}{2 c^{2}(1-\gamma)^{2}\left(t^{\prime}-t\right)}\right)\right\} I_{\nu}\left(\frac{y^{1-\gamma} x^{1-\gamma}}{c^{2}\left(t^{\prime}-t\right)(1-\gamma)^{2}}\right),
$$

where $v^{2}=1 / 4-k_{1}$ and $k_{1}=\left(c^{2} \gamma^{2}-2 c^{2} \gamma-4 m\right) /\left(4 c^{2}(1-\gamma)^{2}\right)$.

Note that if $k=\sqrt{4 m+c^{2}}=0$ so that $m=-c^{2} / 4$, then

$$
A=\frac{x^{2 \gamma-1}}{2}\left[\frac{2 c^{2}}{\ln (x)+D_{1}}+c^{2}\right]
$$

where $D_{1}$ is arbitrary. In this case, the stochastic process (1.4) for $x$ is

$$
d x=c^{2} x^{2 y-1}\left[\frac{1}{\ln (x)+D_{1}}+\frac{1}{2}\right] d t+c x^{\gamma} d X
$$

and the associated TDF for $x$ is

$$
\begin{aligned}
p\left(x, t ; y, t^{\prime}\right)= & y^{1-2 \gamma} \frac{\left(\ln (y)+D_{1}\right)}{\left(\ln (x)+D_{1}\right)} \frac{1}{c^{2}|1-\gamma|\left(t^{\prime}-t\right)} \\
& \times \exp \left\{-\left(\frac{x^{2-2 \gamma}+y^{2-2 \gamma}}{2 c^{2}(1-\gamma)^{2}\left(t^{\prime}-t\right)}\right)\right\} I_{0}\left(\frac{y^{1-\gamma} x^{1-\gamma}}{c^{2}(1-\gamma)^{2}\left(t^{\prime}-t\right)}\right) .
\end{aligned}
$$

This is plotted in Figure 3.1 with $c=0.1, D_{1}=5, x=10, t=0$ for various $\gamma$ and $t^{\prime}$.

3.1.2. $Q=k_{1} / x_{1}^{2}+k_{2} x_{1}^{2}+k_{3}$. For the case corresponding to (2.5b), we require that $A$ satisfy

$$
A^{\prime}-\frac{2 \gamma}{x} A+\frac{1}{c^{2} x^{2 \gamma}} A^{2}=\alpha x^{2 \gamma-2}+\beta x^{2-2 \gamma}+\epsilon
$$

where

$$
\begin{gathered}
\alpha=-k_{1} c^{2}(1-\gamma)^{2}+\frac{c^{2} \gamma^{2}}{4}-\frac{c^{2} \gamma}{2}, \\
\beta=\frac{-k_{2}}{c^{2}(1-\gamma)^{2}}, \\
\epsilon=-k_{3} .
\end{gathered}
$$




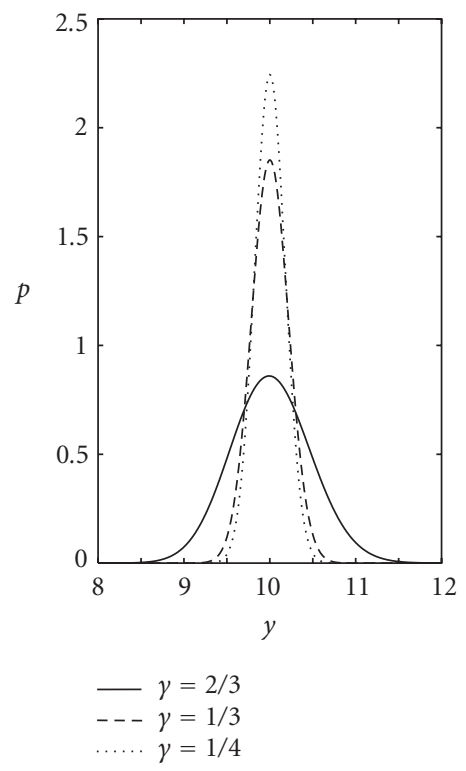

(a)

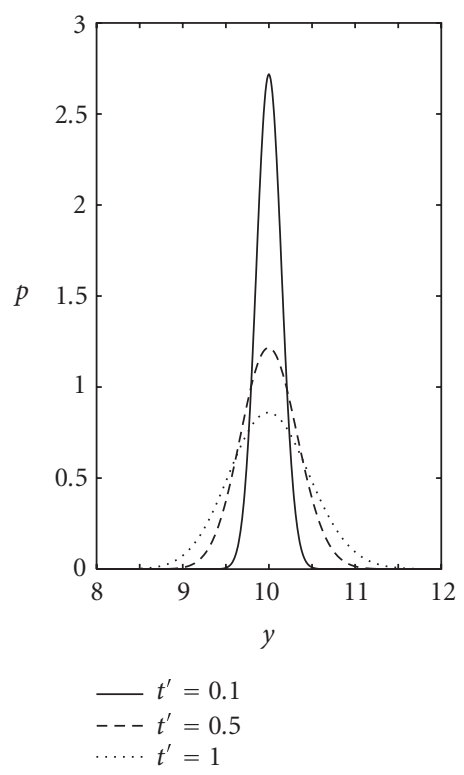

(b)

Figure 3.1. TDFs for the variable $x$ that follows $d x=0.01 x^{2 \gamma-1}[1 /(5+\ln x)+1 / 2] d t+0.1 x^{\gamma} d X$ with $x=10, t=0$ and (a) $\gamma=2 / 3,1 / 3,1 / 4$ at time $t^{\prime}=1$; (b) $\gamma=2 / 3$ at times $t^{\prime}=0.1,0.5,1$.

Then (3.1a)-(3.1c) reduce (1.6a) to

$$
z_{x_{2}}=z_{x_{1} x_{1}}+\left(\frac{k_{1}}{x_{1}^{2}}+k_{2} x_{1}^{2}+k_{3}\right) z
$$

Equation (3.17) can be further reduced to

$$
u_{y_{2}}=u_{y_{1} y_{1}}+\frac{k_{1}}{y_{1}^{2}} u
$$

via a substitution that depends on whether $k_{2}<0$ or $k_{2}>0$. We summarize the results here for the solutions of (1.6a)-(1.6b) and write them in such a way that shows the transformations that were necessary.

Case $1\left(k_{2}<0\right)$. Letting $k_{20}=-k_{2}$, then the solution to $(1.6 \mathrm{a})-(1.6 \mathrm{~b})$ is

$$
p\left(x, t ; y, t^{\prime}\right)=x^{\gamma / 2} \exp \left(\frac{-1}{c^{2}} \int \frac{A(x)}{x^{2 \gamma}} d x\right) z\left(\frac{1}{c(1-\gamma)} x^{1-\gamma}, \frac{t^{\prime}-t}{2}\right),
$$


where

$$
\begin{aligned}
z\left(x_{1}, x_{2}\right)= & \exp \left\{\frac{\sqrt{k_{20}}}{2} x_{1}^{2}+\left(\sqrt{k_{20}}+k_{3}\right) x_{2}\right\} u\left(x_{1} e^{2 \sqrt{k_{20}} x_{2}}, \frac{1}{4 \sqrt{k_{20}}}\left(\exp \left(4 \sqrt{k_{20}} x_{2}\right)-1\right)\right), \\
u\left(y_{1}, y_{2}\right)= & \frac{\sqrt{y_{1}}}{2 y_{2} c^{3 / 2}(1-\gamma)^{1 / 2}} y^{1 / 2-2 \gamma} \exp \left(-\left(\frac{y_{1}^{2} c^{2}(1-\gamma)^{2}+y^{2-2 \gamma}}{4 c^{2}(1-\gamma)^{2} y_{2}}\right)\right) I_{\nu}\left(\frac{y^{1-\gamma} y_{1}}{2 y_{2} c(1-\gamma)}\right) \\
& \times \exp \left(\frac{1}{c^{2}} \int^{y} \frac{A(x)}{x^{2 \gamma}} d x\right) \exp \left(-\frac{\sqrt{k_{20}}}{2} \frac{y^{2-2 \gamma}}{c^{2}(1-\gamma)^{2}}\right)
\end{aligned}
$$

where $v^{2}=1 / 4-k_{1}$.

Case $2\left(k_{2}>0\right)$. For $k_{2}>0$, the solution to $(1.6 \mathrm{a})-(1.6 \mathrm{~b})$ is

$$
p\left(x, t ; y, t^{\prime}\right)=x^{\gamma / 2} \exp \left(\frac{-1}{c^{2}} \int \frac{A(x)}{x^{2 \gamma}} d x\right) z\left(\frac{1}{c(1-\gamma)} x^{1-\gamma}, \frac{t^{\prime}-t}{2}\right)
$$

where

$$
\begin{aligned}
z\left(x_{1}, x_{2}\right)= & \frac{1}{\sqrt{\left|\cos \left(2 \sqrt{k_{2}} x_{2}\right)\right|}} \exp \left[\frac{\sqrt{k_{2}}}{2} x_{1}^{2} \tan \left(2 \sqrt{k_{2}} x_{2}\right)+k_{3} x_{2}\right] \\
& \times u\left(\frac{x_{1}}{\cos \left(2 \sqrt{k_{2}} x_{2}\right)}, \frac{1}{2 \sqrt{k_{2}}} \tan \left(2 \sqrt{k_{2}} x_{2}\right)\right), \\
u\left(y_{1}, y_{2}\right)= & \frac{y^{1 / 2-2 \gamma}}{c^{3 / 2}(1-\gamma)^{1 / 2}} \frac{\sqrt{y_{1}}}{2 y_{2}} \exp \left\{-\left(\frac{y_{1} 2 c^{2}(1-\gamma)^{2}+y^{2-2 \gamma}}{4 c^{2}(1-\gamma)^{2} y_{2}}\right)\right\} I_{\nu}\left(\frac{y^{1-\gamma} y_{1}}{2 y_{2} c(1-\gamma)}\right) \\
& \times \exp \left(\frac{1}{c^{2}} \int^{y} \frac{A(x)}{x^{2 \gamma}} d x\right),
\end{aligned}
$$

where $v^{2}=1 / 4-k_{1}$.

Note that in general for $\gamma \geq 0, \gamma \neq 1$, that in the expanded expressions for $p$ from (3.19) and (3.21), the coefficient $1 /(1-\gamma)$ should be replaced by $1 /|1-\gamma|$.

Example 3.2. With $\gamma=3 / 2$ so that $B=c x^{3 / 2}, \alpha=\epsilon=0$, a solution to (3.16a)-(3.16d) is

$$
A=c^{2} x^{2}\left[1+\frac{\sqrt{a}}{x} \frac{C_{1} \sin (\sqrt{a} / x)+C_{2} \cos (\sqrt{a} / x)}{C_{1} \cos (\sqrt{a} / x)-C_{2} \sin (\sqrt{a} / x)}\right]
$$

where $a=-\beta / c^{2}$ and $\beta, C_{1}$, and $C_{2}$ are arbitrary constants. 
In this case, $\beta<0$ and so $k_{2}=-\beta c^{2}(1-\gamma)^{2}>0$ and $k_{3}=0, k_{1}=-3 / 4, a=-\beta / c^{2}$. Hence we find the fundamental solution

$$
\begin{aligned}
p\left(x, t ; y, t^{\prime}\right)= & \frac{\sqrt{-\beta}}{c \sqrt{x} y^{3 / 2}} \frac{\left(C_{1} \cos (\sqrt{a} / y)-C_{2} \sin (\sqrt{a} / y)\right)}{\left(C_{1} \cos (\sqrt{a} / x)-C_{2} \sin (\sqrt{a} / x)\right)} \\
& \times \exp \left\{\frac{\sqrt{-\beta}}{c}\left[\frac{y \sin ^{2}\left(\sqrt{k_{2}}\left(t^{\prime}-t\right)\right)-y-x \cos ^{2}\left(\sqrt{k_{2}}\left(t^{\prime}-t\right)\right)}{x y \cos \left(\sqrt{k_{2}}\left(t^{\prime}-t\right)\right) \sin \left(\sqrt{k_{2}}\left(t^{\prime}-t\right)\right)}\right]\right\} \\
& \times I_{1}\left(\frac{2 \sqrt{-\beta}}{c \sqrt{x y} \sin \left(\sqrt{k_{2}}\left(t^{\prime}-t\right)\right)}\right) \times \frac{1}{\sin \left(\sqrt{-\beta c^{2}}\left(t^{\prime}-t\right) / 2\right)} .
\end{aligned}
$$

Example 3.3. We note that in the case where $A(x)=\mu x$ and $0 \leq \gamma<1$, the density function has previously been found by Feller $[11,12]$ and this then has been used to find option prices via risk-neutral valuation as described in the introduction (see Cox and Ross [8]).

The same result can be found here with

$$
\alpha=0, \quad \beta=\frac{\mu^{2}}{c^{2}}, \quad \epsilon=\mu(1-2 \gamma)
$$

in (3.16a) so that from $(3.16 \mathrm{~b})-(3.16 \mathrm{~d})$,

$$
\begin{gathered}
k_{1}=\frac{\gamma(\gamma-2)}{4(1-\gamma)^{2}}, \\
k_{2}=-(1-\gamma)^{2} \mu^{2}<0, \\
k_{3}=-\mu(1-2 \gamma) .
\end{gathered}
$$

From (3.19)-(3.20), we get

$$
\begin{aligned}
p\left(x, t ; y, t^{\prime}\right)= & \frac{2 \mu}{c^{2}} \frac{x^{1 / 2} y^{1 / 2-2 \gamma} e^{(\mu / 2)\left(t^{\prime}-t\right)}}{\left(e^{2(1-\gamma) \mu\left(t^{\prime}-t\right)}-1\right)} \exp \left\{\frac{-\mu}{c^{2}(1-\gamma)}\left(\frac{x^{2-2 \gamma} e^{2(1-\gamma) \mu\left(t^{\prime}-t\right)}+y^{2-2 \gamma}}{e^{2(1-\gamma) \mu\left(t^{\prime}-t\right)}-1}\right)\right\} \\
& \times I_{1 / 2(1-\gamma)}\left\{\frac{2 y^{1-\gamma} \mu x^{1-\gamma} e^{(1-\gamma) \mu\left(t^{\prime}-t\right)}}{c^{2}(1-\gamma)\left[e^{2(1-\gamma) \mu\left(t^{\prime}-t\right)}-1\right]}\right\},
\end{aligned}
$$

which agrees with the result in [8].

3.1.3. $Q=k_{1} x_{1}^{2}+k_{2} x+k_{3}$. For the case corresponding to (2.5c), we require that $A$ satisfy

$$
A^{\prime}-\frac{2 \gamma}{x} A+\frac{1}{c^{2} x^{2 \gamma}} A^{2}=\alpha x^{2-2 \gamma}+\beta x^{1-\gamma}+\epsilon+m x^{2 \gamma-2}
$$


12 Fundamental solutions to Kolmogorov equations

where

$$
\begin{gathered}
\alpha=\frac{-k_{1}}{c^{2}(1-\gamma)^{2}}, \\
\beta=\frac{-k_{2}}{c(1-\gamma)}, \\
\epsilon=-k_{3}, \\
m=\frac{c^{2} \gamma^{2}}{4}-\frac{c^{2} \gamma}{2} .
\end{gathered}
$$

Then (3.1a)-(3.1c) reduce (1.6a) to

$$
z_{x_{2}}=z_{x_{1} x_{1}}+\left(k_{1} x_{1}^{2}+k_{2} x_{1}+k_{3}\right) z
$$

Equation (3.29) is always reducible to the form

$$
w_{y_{2}}=w_{y_{1} y_{1}}+k w
$$

where $k$ is some constant. However, the transformation necessary to get this reduction depends on the coefficient $k_{1}$. We consider the different cases and summarize the results below.

Case $1\left(k_{1}=0\right)$. In this case, $k=k_{3}$ in (3.30) and the solution to (1.6a)-(1.6b) is

$$
p\left(x, t ; y, t^{\prime}\right)=x^{\gamma / 2} \exp \left(\frac{-1}{c^{2}} \int \frac{A(x)}{x^{2 \gamma}} d x\right) z\left(\frac{1}{c(1-\gamma)} x^{1-\gamma}, \frac{t^{\prime}-t}{2}\right)
$$

where

$$
\begin{gathered}
z\left(x_{1}, x_{2}\right)=\exp \left(k_{2} x_{1} x_{2}+\frac{k_{2}^{2} x_{2}^{3}}{3}\right) w\left(x_{1}+k_{2} x_{2}^{2}, x_{2}\right), \\
w\left(y_{1}, y_{2}\right)=\frac{y^{-(3 / 2) \gamma}}{2 c \sqrt{\pi y_{2}}} \exp \left(\frac{1}{c^{2}} \int^{y} \frac{A(x)}{x^{2 \gamma}} d x\right) \exp \left\{\frac{-\left(y_{1} c(1-\gamma)-y^{1-\gamma}\right)^{2}}{4 y_{2} c^{2}(1-\gamma)^{2}}+k_{3} y_{2}\right\} .
\end{gathered}
$$

Case $2\left(k_{1}<0\right)$. We let $k_{1}=-\lambda^{2}$.

For this case, $k=0$ in (3.30) and the solution to (1.6a)-(1.6b) is

$$
p\left(x, t ; y, t^{\prime}\right)=x^{\gamma / 2} \exp \left(-\frac{1}{c^{2}} \int \frac{A(x)}{x^{2 \gamma}} d x\right) z\left(\frac{1}{c(1-\gamma)} x^{1-\gamma}, \frac{t^{\prime}-t}{2}\right)
$$


where

$$
\begin{aligned}
z\left(x_{1}, x_{2}\right)= & \exp \left\{\frac{\lambda}{2} x_{1}^{2}-\frac{k_{2}}{2 \lambda} x_{1}+\left(\lambda+k_{3}+\frac{k_{2}^{2}}{4 \lambda^{2}}\right) x_{2}\right\} \\
& \times w\left(x_{1} e^{2 \lambda x_{2}}-\frac{k_{2}}{2 \lambda^{2}} e^{2 \lambda x_{2}}, \frac{1}{4 \lambda}\left(e^{4 \lambda x_{2}}-1\right)\right), \\
w\left(y_{1}, y_{2}\right)= & \frac{y^{-3 \gamma / 2}}{2 c \sqrt{\pi y_{2}}} \exp \left\{-\frac{\left(y_{1}+k_{2} / 2 \lambda^{2}-y^{1-\gamma / c}(1-\gamma)\right)^{2}}{4 y_{2}}-\frac{\lambda}{2} \frac{y^{2-2 \gamma}}{c^{2}(1-\gamma)^{2}}+\frac{k_{2}}{2 \lambda} \frac{y^{1-\gamma}}{c(1-\gamma)}\right\} \\
& \times \exp \left(\frac{1}{c^{2}} \int^{y} \frac{A(x)}{x^{2 \gamma}} d x\right) .
\end{aligned}
$$

Case $3\left(k_{1}>0\right)$. We let $k_{1}=\lambda^{2}$.

In this case, $k=-k_{2}^{2} / 8 \lambda^{2}$ in (3.30) and the solution to (1.6a)-(1.6b) is

$$
p\left(x, t ; y, t^{\prime}\right)=x^{\gamma / 2} \exp \left(-\frac{1}{c^{2}} \int \frac{A(x)}{x^{2 \gamma}} d x\right) z\left(\frac{1}{c(1-\gamma)} x^{1-\gamma}, \frac{t^{\prime}-t}{2}\right),
$$

where

$$
\begin{aligned}
z\left(x_{1}, x_{2}\right)= & \frac{1}{\sqrt{\left|\cos 2 \lambda x_{2}\right|}} \exp \left\{\frac{\lambda}{2} x_{1}^{2} \tan \left(2 \lambda x_{2}\right)+\frac{k_{2}}{2 \lambda} x_{1} \tan \left(2 \lambda x_{2}\right)+\left(k_{3}-\frac{k_{2}^{2}}{4 \lambda^{2}}\right) x_{2}\right\} \\
& \times \exp \left\{\frac{3 k_{2}^{2}}{16 \lambda^{3}} \tan \left(2 \lambda x_{2}\right)\right\} w\left(\left(x_{1}+\frac{k_{2}}{2 \lambda^{2}}\right) \sec \left(2 \lambda x_{2}\right), \frac{1}{2 \lambda} \tan \left(2 \lambda x_{2}\right)\right), \\
w\left(y_{1}, y_{2}\right)= & \frac{y^{-3 \gamma / 2}}{2 c \sqrt{\pi y_{2}}} \exp \left\{\frac{-\left(y_{1}-y^{(1-\gamma)} / c(1-\gamma)-k_{2} / 2 \lambda^{2}\right)^{2}}{4 y_{2}}-\frac{k_{2}^{2}}{8 \lambda^{2}} y_{2}\right\} \\
& \times \exp \left(\frac{1}{c^{2}} \int^{y} \frac{A(x)}{x^{2 \gamma}} d x\right) .
\end{aligned}
$$

Example 3.4. A solution to (3.28) with

$$
\alpha=\frac{\mu^{2}}{c^{2}}, \quad \beta=0, \quad \epsilon=\mu(1-2 \gamma)+\frac{2 \mu r}{c^{2}}, \quad m=-r+\frac{r^{2}}{c^{2}}=\frac{c^{2} \gamma^{2}}{4}-\frac{c^{2} \gamma}{2}
$$

is $A=\mu x+r x^{2 \gamma-1}$, where

$$
r=\frac{(2-\gamma) c^{2}}{2} \text { or } \quad r=\frac{\gamma c^{2}}{2}
$$

and where $\mu$ is arbitrary.

The corresponding stochastic differential equation (1.4) for $x$ is

$$
d x=\left(r x^{2 \gamma-1}+\mu x\right) d t+c x^{\gamma} d X
$$


14 Fundamental solutions to Kolmogorov equations

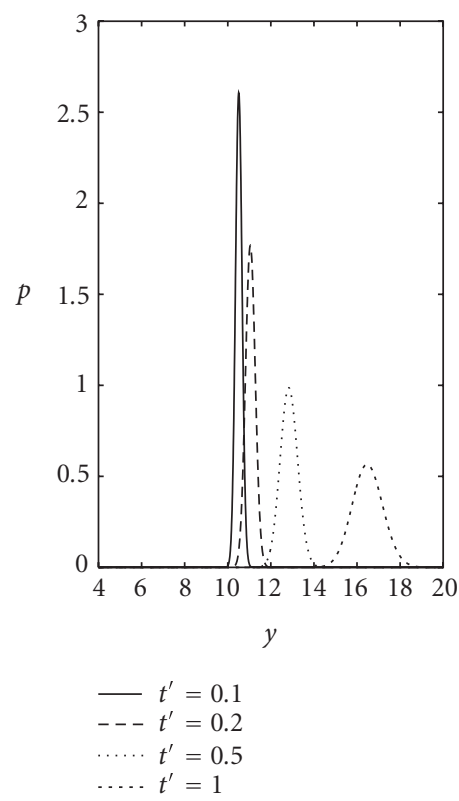

(a)

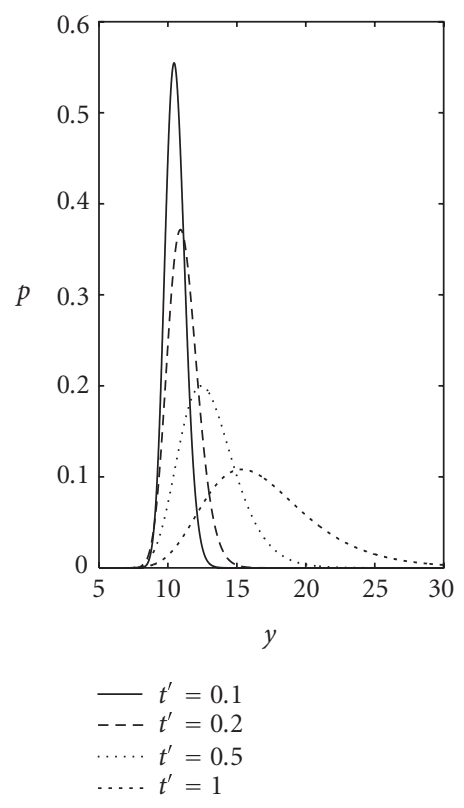

(b)

Figure 3.2. TDFs for the variable $x$ which follows (a) $d x=\left(0.003 x^{1 / 3}+0.5 x\right) d t+0.1 x^{2 / 3} d X$, (b) $d x=$ $\left(0.006 x^{5 / 3}+0.5 x\right) d t+0.1 x^{4 / 3} d X$, with $x=10, t=0$ at times $t^{\prime}=0.1,0.2,0.5,1$.

with $r$ given in (3.38). The TDF for $x$ with $r=\gamma c^{2} / 2$ is

$p\left(x, t ; y, t^{\prime}\right)=\frac{1}{c y^{\gamma} \sqrt{\pi}} \sqrt{\frac{(1-\gamma) \mu}{\exp \left\{2 \mu\left(t^{\prime}-t\right)(1-\gamma)\right\}-1}} \exp \left\{\frac{-\mu}{c^{2}(1-\gamma)} \frac{\left(x^{1-\gamma} e^{\mu(1-\gamma)\left(t^{\prime}-t\right)}-y^{1-\gamma}\right)^{2}}{\left(e^{2 \mu\left(t^{\prime}-t\right)(1-\gamma)}-1\right)}\right\}$,

and when $r=(2-\gamma) c^{2} / 2$, the TDF for $x$ is

$$
\begin{aligned}
p\left(x, t ; y, t^{\prime}\right)= & \frac{x^{\gamma-1} y^{1-2 \gamma}}{c \sqrt{\pi}} \sqrt{\frac{(1-\gamma) \mu}{\exp \left\{2 \mu\left(t^{\prime}-t\right)(1-\gamma)\right\}-1}} \\
& \times \exp \left\{\frac{-\mu}{c^{2}(1-\gamma)} \frac{\left(x^{1-\gamma} e^{\mu(1-\gamma)\left(t^{\prime}-t\right)}-y^{1-\gamma}\right)^{2}}{\left(e^{2 \mu\left(t^{\prime}-t\right)(1-\gamma)}-1\right)}\right\} \exp \left\{-\mu(1-\gamma)\left(t^{\prime}-t\right)\right\} .
\end{aligned}
$$

Sample plots of the TDF $p\left(x, t ; y, t^{\prime}\right)$ are given in Figure 3.2, where $c=0.1, \mu=0.5$, and $r=\gamma c^{2} / 2$ for $\gamma=2 / 3$ and $\gamma=4 / 3$. 
3.2. $A=A(x, t)$. For $A=A(x, t)$, with $B=c x^{\gamma}$, from (2.3d),

$$
A=c^{2} x^{2 \gamma-1}\left(\frac{\gamma}{2}+\frac{\alpha^{\prime}(x)}{\alpha(x)} x+k^{\prime}(x) x t\right) .
$$

With $x$ defined in terms of $x_{1}$ as given by (3.1a), $x_{2}=t / 2$ and $z$ as given by $(2.3 \mathrm{c})$ and $(2.3 \mathrm{~d}),(1.6 \mathrm{a})$ reduces to

$$
z_{x_{2}}+z_{x_{1} x_{1}}+Q\left(x_{1}, x_{2}\right) z=0
$$

where

$$
\begin{aligned}
Q= & -c^{2} x^{2 \gamma-1}\left[\gamma k^{\prime}(x)+x k^{\prime \prime}(x)+\frac{2 \alpha^{\prime}(x)}{\alpha(x)} x k^{\prime}(x)\right] t-c^{2} x^{2 \gamma}\left(k^{\prime}(x)\right)^{2} t^{2} \\
& -c^{2}\left(x^{2 \gamma-1} \gamma \frac{\alpha^{\prime}(x)}{\alpha(x)}+x^{2 \gamma} \frac{\alpha^{\prime \prime}(x)}{\alpha(x)}\right)-2 k(x) .
\end{aligned}
$$

With (3.44), (3.43) will be reducible to a constant coefficient equation when

$$
Q=\frac{f_{1}(t) x^{2-2 \gamma}}{c^{2}(1-\gamma)^{2}}+\frac{f_{2}(t) x^{1-\gamma}}{c(1-\gamma)}+f_{3}(t)
$$

that is, a quadratic in $x_{1}$.

Comparing (3.44) and (3.45), we find that this will occur when $k(x)=\beta x^{1-\gamma}$ and when $k(x)=\beta x^{2-2 \gamma}$, where $\beta$ is constant. With $k(x)=\beta x^{1-\gamma}$, admissible functions for $\alpha(x)$, so that $Q$ is a quadratic in $x_{1}$, are $\alpha(x)=e^{(\epsilon /(1-\gamma)) x^{1-\gamma}}$ and $\alpha(x)=e^{(\epsilon /(2-2 \gamma)) x^{2-2 \gamma}}$, where $\epsilon$ is constant. These together with $\alpha(x)=x^{1-\gamma}$ are also admissible functions for $k(x)=\beta x^{2-2 \gamma}$. In all of these cases, (3.43) is further reducible to the classical heat equation. We now summarize the results with the fundamental solutions of (1.6a) written in such a way that shows the transformations that were necessary for reduction to the classical heat equation.

3.2.1. $k(x)=\beta x^{1-\gamma}$.

Case 1. $\alpha(x)=\exp \left((\epsilon /(1-\gamma)) x^{1-\gamma}\right)$.

This case corresponds to the stochastic differential equation

$$
d x=c^{2}\left(\frac{\gamma}{2} x^{2 \gamma-1}+\epsilon x^{\gamma}+\beta(1-\gamma) x^{\gamma} t\right) d t+c x^{\gamma} d X
$$

for $x$. The solution to (1.6a)-(1.6b) is

$$
p\left(x, t ; y, t^{\prime}\right)=\exp \left\{\frac{-\epsilon}{1-\gamma} x^{1-\gamma}-\beta x^{1-\gamma} t\right\} z\left(\frac{x^{1-\gamma}}{c(1-\gamma)}, \frac{t}{2}\right),
$$

where

$$
\begin{aligned}
z\left(x_{1}, x_{2}\right)= & \exp \left\{2 \beta c(1-\gamma) x_{1} x_{2}+c^{2} \epsilon^{2} x_{2}+2 c^{2} \epsilon \beta(1-\gamma) x_{2}^{2}\right\} \\
& \times w\left(x_{1}-2 \beta c(1-\gamma) x_{2}^{2}, \frac{t^{\prime}}{2}-x_{2}\right),
\end{aligned}
$$


16 Fundamental solutions to Kolmogorov equations

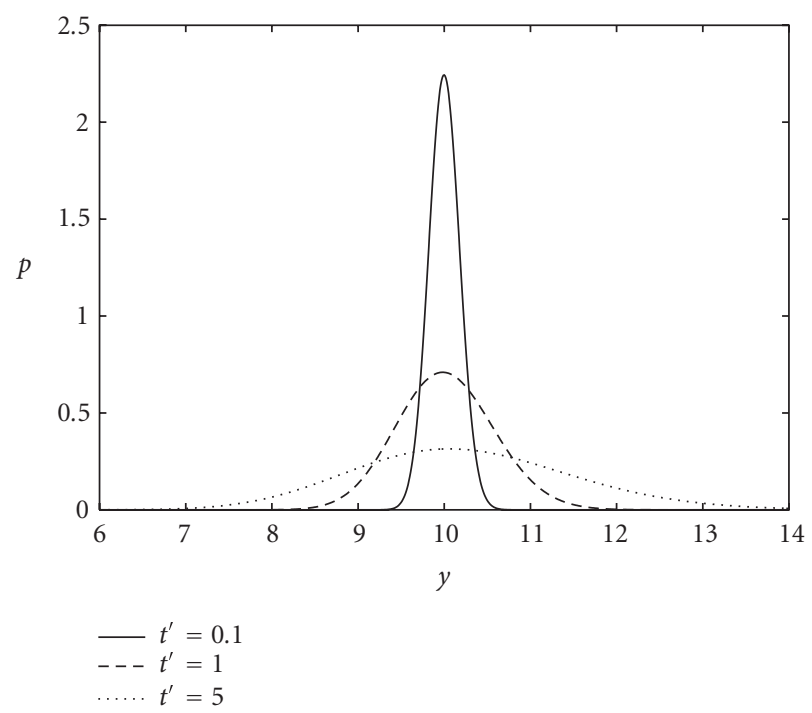

Figure 3.3. TDFs for the variable $x$ which follows $d x=0.01 x^{3 / 4}\left[t / 4+(3 / 8) x^{-1 / 4}\right] d t+0.1 x^{3 / 4} d X$ with $x=10, t=0$ at times $t^{\prime}=0.1,1,5$.

and where

$$
\begin{aligned}
w\left(y_{1}, y_{2}\right)= & \frac{y^{-\gamma}}{2 c \sqrt{\pi y_{2}}} \exp \left\{\frac{-\left(y_{1}-y^{1-\gamma} / c(1-\gamma)+c \beta(1-\gamma)\left(t^{\prime}\right)^{2} / 2\right)^{2}}{4 y_{2}}\right\} \\
& \times \exp \left\{\frac{\epsilon y^{1-\gamma}}{c(1-\gamma)}-\frac{c^{2} \epsilon^{2} t^{\prime}}{2}-c^{2} \epsilon \beta(1-\gamma) \frac{\left(t^{\prime}\right)^{2}}{2}\right\} .
\end{aligned}
$$

Example 3.5. With $\beta=1, \epsilon=0$, the above solution can be simplified to

$$
p\left(x, t ; y, t^{\prime}\right)=\frac{y^{-\gamma}}{c \sqrt{\pi} \sqrt{2\left(t^{\prime}-t\right)}} \exp \left\{\frac{-\left(-2 x^{1-\gamma}-c^{2}(1-\gamma)^{2}\left(\left(t^{\prime}\right)^{2}-t^{2}\right)+2 y^{1-\gamma}\right)^{2}}{8 c^{2}(1-\gamma)^{2}\left(t^{\prime}-t\right)}\right\} .
$$

This is plotted in Figure 3.3 with $x=10, \gamma=3 / 4, c=0.1$. 
Case 2. $\alpha(x)=\exp \left((\epsilon /(2-2 \gamma)) x^{2-2 \gamma}\right), \epsilon \neq 0$.

This case corresponds to the stochastic differential equation

$$
d x=c^{2}\left(\frac{\gamma}{2} x^{2 \gamma-1}+\epsilon x+\beta(1-\gamma) x^{\gamma} t\right) d t+c x^{\gamma} d X
$$

for $x$.

The solution to $(1.6 \mathrm{a})-(1.6 \mathrm{~b})$ is

$$
p\left(x, t ; y, t^{\prime}\right)=\exp \left(\frac{-\epsilon}{2-2 \gamma} x^{2-2 \gamma}-\beta x^{1-\gamma} t\right) z\left(\frac{1}{c(1-\gamma)} x^{1-\gamma}, \frac{t}{2}\right)
$$

where

$$
\begin{aligned}
z\left(x_{1}, x_{2}\right) & \\
= & \exp \left\{\frac{c^{2} \epsilon(1-\gamma)}{2} x_{1}^{2}+2 c \beta(1-\gamma) x_{1} x_{2}\right\} \\
& \times w\left(\left(x_{1}+\frac{2 \beta}{c \epsilon} x_{2}+\frac{\beta}{c^{3} \epsilon^{2}(1-\gamma)}\right) e^{-2 c^{2} \epsilon(1-\gamma) x_{2}}, \frac{1}{4 c^{2} \epsilon(1-\gamma)}\left(e^{-4 c^{2} \epsilon(1-\gamma) x_{2}}-e^{-2 c^{2} \epsilon(1-\gamma) t^{\prime}}\right)\right), \\
w\left(y_{1}, y_{2}\right) & \\
= & \frac{y^{-\gamma}}{2 c \sqrt{\pi y_{2}}} \exp \left(-c^{2} \epsilon(1-\gamma) t^{\prime}\right) \\
& \times \exp \left[\frac{-\left(y_{1}-\left\{y^{1-\gamma / c}(1-\gamma)+\beta t^{\prime} / c \epsilon+\beta / c^{3} \epsilon^{2}(1-\gamma)\right\} e^{-c^{2} \epsilon(1-\gamma) t^{\prime}}\right)^{2}}{4 y_{2}}\right] .
\end{aligned}
$$

3.2.2. $k(x)=\beta x^{2-2 \gamma}, \beta>0$.

Case 1. $\alpha(x)=\exp \left((\epsilon /(1-\gamma)) x^{1-\gamma}\right)$.

The corresponding stochastic differential equation for $x$ is

$$
d x=c^{2}\left(\frac{\gamma}{2} x^{2 \gamma-1}+\epsilon x^{\gamma}+\beta(2-2 \gamma) x t\right) d t+c x^{\gamma} d X
$$

The solution to (1.6a)-(1.6b) is

$$
p\left(x, t ; y, t^{\prime}\right)=\exp \left(\frac{-\epsilon}{1-\gamma} x^{1-\gamma}-\beta x^{2-2 \gamma} t\right) z\left(\frac{1}{c(1-\gamma)} x^{1-\gamma}, \frac{t}{2}\right),
$$


where

$$
\begin{aligned}
z\left(x_{1}, x_{2}\right)= & \exp \left\{2 r x_{1}^{2} x_{2}+c \epsilon x_{1}\right\} \\
\times & w\left(x_{1} e^{-4 r x_{2}^{2}}-\frac{\epsilon \sqrt{\pi}}{2 \sqrt{\beta(1-\gamma)^{2}}} \operatorname{erf}\left(2 \sqrt{r} x_{2}\right), \frac{\sqrt{\pi}}{4 \sqrt{2 r}}\left[\operatorname{erf}\left(\sqrt{2 r} t^{\prime}\right)-\operatorname{erf}\left(\sqrt{8 r} x_{2}\right)\right]\right), \\
w\left(y_{1}, y_{2}\right) & \\
= & \frac{y^{-\gamma}}{2 c \sqrt{\pi y_{2}}} \exp \left\{-r\left(t^{\prime}\right)^{2}\right\} \\
& \times \exp \left\{\frac{-\left[y_{1}-\left(y^{1-\gamma / c}(1-\gamma)\right) e^{-r\left(t^{\prime}\right)^{2}}+(c \epsilon \sqrt{\pi} / 2 \sqrt{r}) \operatorname{erf}\left(\sqrt{r} t^{\prime}\right)\right]^{2}}{4 y_{2}}\right\},
\end{aligned}
$$

and where $r=c^{2} \beta(1-\gamma)^{2}$.

Example 3.6. With $\beta=1, \epsilon=0$, the TDF can be simplified to

$$
\begin{aligned}
p\left(x, t ; y, t^{\prime}\right)= & \frac{y^{-\gamma} 2^{1 / 4}}{c \pi^{3 / 4}} \sqrt{\frac{c(\gamma-1)}{\operatorname{erf}\left(\sqrt{2} c(\gamma-1) t^{\prime}\right)-\operatorname{erf}(\sqrt{2} c(\gamma-1) t)}} \\
& \times \exp \left\{\frac{\sqrt{2}\left(x^{1-\gamma} e^{-c^{2}(1-\gamma)^{2} t^{2}}-y^{1-\gamma} e^{-c^{2}(1-\gamma)^{2}\left(t^{\prime}\right)^{2}}\right)^{2}}{(1-\gamma) \sqrt{\pi} c\left[\operatorname{erf}\left(\sqrt{2} c(\gamma-1) t^{\prime}\right)-\operatorname{erf}(\sqrt{2} c(\gamma-1) t)\right]}\right\} \\
& \times \exp \left\{-(1-\gamma)^{2} c^{2}\left(t^{\prime}\right)^{2}\right\}
\end{aligned}
$$

This TDF is plotted in Figure 3.4 with $x=10, c=0.1, \gamma=1 / 4$.

Case 2. $\alpha(x)=\exp \left((\epsilon /(2-2 \gamma)) x^{2-2 \gamma}\right)$.

The corresponding stochastic differential equation for $x$ is

$$
d x=c^{2}\left(\frac{\gamma}{2} x^{2 \gamma-1}+\epsilon x+\beta(2-2 \gamma) x t\right) d t+c x^{\gamma} d X
$$

The solution to $(1.6 \mathrm{a})-(1.6 \mathrm{~b})$ is

$$
p\left(x, t ; y, t^{\prime}\right)=\exp \left(\frac{-\epsilon}{2-2 \gamma} x^{2-2 \gamma}-\beta x^{2-2 \gamma} t\right) z\left(\frac{1}{c(1-\gamma)} x^{1-\gamma}, \frac{t}{2}\right),
$$




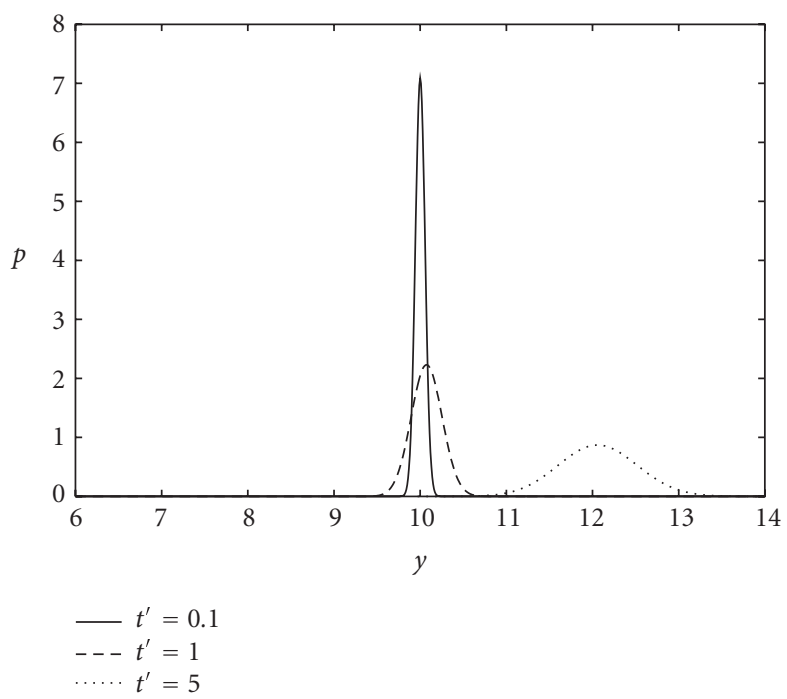

Figure 3.4. TDFs for the variable $x$ which follows $d x=0.01 x\left[(3 / 2) t+(1 / 8) x^{-3 / 2}\right] d t+0.1 x^{1 / 4} d X$ with $x=10, t=0$ at times $t^{\prime}=0.1,1,5$.

where

$$
\begin{aligned}
z\left(x_{1}, x_{2}\right)= & \exp \left\{2 r x_{1}^{2} x_{2}+\frac{x_{1}^{2}}{2} \epsilon c^{2}(1-\gamma)\right\} \\
& \times w\left(x_{1} \exp \left(-4 r\left(x_{2}+n\right)^{2}\right), \frac{\sqrt{\pi}}{4 \sqrt{2 r}}\left\{\operatorname{erf}\left(2 \sqrt{2 r}\left(\frac{t^{\prime}}{2}+n\right)\right)-\operatorname{erf}\left(2 \sqrt{2 r}\left(x_{2}+n\right)\right)\right\}\right), \\
w\left(y_{1}, y_{2}\right)= & \frac{y^{-\gamma}}{2 c \sqrt{\pi y_{2}}} \exp \left(-4 r\left(\frac{t^{\prime}}{2}+n\right)^{2}\right) \exp \left\{\frac{-\left[y_{1}-y^{1-\gamma / c}(1-\gamma) e^{-4 r\left(t^{\prime} / 2+n\right)^{2}}\right]^{2}}{4 y_{2}}\right\},
\end{aligned}
$$

and where $r=\beta c^{2}(1-\gamma)^{2}$ and $n=\epsilon / 4 \beta(1-\gamma)$.

Case 3. $\alpha(x)=x^{1-\gamma}$.

The corresponding stochastic differential equation for $x$ is

$$
d x=c^{2}\left(x^{2 \gamma-1}\left(1-\frac{\gamma}{2}\right)+\beta(2-2 \gamma) x t\right) d t+c x^{\gamma} d X
$$

The solution to $(1.6 \mathrm{a})-(1.6 \mathrm{~b})$ is

$$
p\left(x, t ; y, t^{\prime}\right)=x^{\gamma-1} \exp \left(-\beta x^{2-2 \gamma} t\right) z\left(\frac{1}{c(1-\gamma)} x^{1-\gamma}, \frac{t}{2}\right)
$$


where

$$
\begin{gathered}
z\left(x_{1}, x_{2}\right)=\exp \left(2 r x_{1}^{2} x_{2}+4 r x_{2}^{2}\right) w\left(x_{1} e^{-4 r x_{2}^{2}}, \frac{\sqrt{\pi}}{4 \sqrt{2 r}}\left[\operatorname{erf}\left(\sqrt{2 r} t^{\prime}\right)-\operatorname{erf}\left(2 \sqrt{2 r} x_{2}\right)\right]\right), \\
w\left(y_{1}, y_{2}\right)=\frac{y^{1-2 \gamma}}{2 c \sqrt{\pi y_{2}}} \exp \left(-r\left(t^{\prime}\right)^{2}\right) \exp \left\{\frac{-\left(y_{1}-\left(y^{1-\gamma / c}(1-\gamma)\right) e^{-r\left(t^{\prime}\right)^{2}}\right)^{2}}{4 y_{2}}\right\}
\end{gathered}
$$

with $r=\beta c^{2}(1-\gamma)^{2}$.

4. $B(x)=\left(\alpha_{1}+\alpha_{2} x+\alpha_{3} x^{2}\right)^{1 / 2}$.

For $A=A(x, t)$ and $B(x)=\left(\alpha_{1}+\alpha_{2} x+\alpha_{3} x^{2}\right)^{1 / 2}$, the transformation

$$
\begin{gathered}
x_{1}=\int \frac{1}{\sqrt{\alpha_{1}+\alpha_{2} x+\alpha_{3} x^{2}}} d x, \\
x_{2}=\frac{t}{2} \\
z=\alpha(x) e^{k(x) t} p
\end{gathered}
$$

subject to (2.3d) transforms (1.6a) to

$$
z_{x_{2}}+z_{x_{1} x_{1}}+Q\left(x_{1}, x_{2}\right) z=0
$$

where

$$
\begin{aligned}
Q= & -\left(k^{\prime}(x)\right)^{2}\left(\alpha_{1}+\alpha_{2} x+\alpha_{3} x^{2}\right) t^{2} \\
& -\left[\left(k^{\prime \prime}(x)+2 k^{\prime}(x) \frac{\alpha^{\prime}(x)}{\alpha(x)}\right)\left(\alpha_{1}+\alpha_{2} x+\alpha_{3} x^{2}\right)+\frac{k^{\prime}(x)}{2}\left(\alpha_{2}+2 \alpha_{3} x\right)\right] t \\
& -\left[\frac{\alpha^{\prime \prime}(x)}{\alpha(x)}\left(\alpha_{1}+\alpha_{2} x+\alpha_{3} x^{2}\right)+2 k(x)+\frac{\alpha^{\prime}(x)}{2 \alpha(x)}\left(\alpha_{2}+2 \alpha_{3} x\right)\right]
\end{aligned}
$$

in which we have used from $(2.3 \mathrm{~d})$ that

$$
A=k^{\prime}(x) t\left(\alpha_{1}+\alpha_{2} x+\alpha_{3} x^{2}\right)+\frac{\alpha^{\prime}(x)}{\alpha(x)}\left(\alpha_{1}+\alpha_{2} x+\alpha_{3} x^{2}\right)+\frac{1}{4}\left(\alpha_{2}+2 \alpha_{3} x\right)
$$

In turn, (4.2) with (4.3) is reducible to a constant coefficient equation when $Q$ is a quadratic in $x_{1}$. This can be achieved when $k(x)$ is a multiple of $x_{1}$, and $\alpha(x)$ takes one of the two forms as considered below. In both cases, $(4.2)$ is further reducible to the classical heat equation. The results are summarized below. 
Case 1. $k(x)=\beta x_{1}, \alpha(x)=\exp \left\{\epsilon \int\left(d x /\left(\sqrt{\alpha_{1}+\alpha_{2} x+\alpha_{3} x^{2}}\right)\right)\right\}$.

The corresponding stochastic differential equation for $x$ is

$$
d x=\left[(\beta t+\epsilon) \sqrt{\alpha_{1}+\alpha_{2} x+\alpha_{3} x^{2}}+\frac{1}{4}\left(\alpha_{2}+2 \alpha_{3} x\right)\right] d t+\sqrt{\alpha_{1}+\alpha_{2} x+\alpha_{3} x^{2}} d X .
$$

The solution to (1.6a)-(1.6b) is

$$
p\left(x, t ; y, t^{\prime}\right)=\exp \left(-(\epsilon+\beta t) \int \frac{1}{\sqrt{\alpha_{1}+\alpha_{2} x+\alpha_{3} x^{2}}} d x\right) z\left(\int \frac{1}{\sqrt{\alpha_{1}+\alpha_{2} x+\alpha_{3} x^{2}}} d x, \frac{t}{2}\right)
$$

where

$$
\begin{aligned}
z\left(x_{1}, x_{2}\right)= & \exp \left(2 \beta x_{1} x_{2}+\frac{4}{3} \beta(1-\beta) x_{2}^{3}+2 \beta \epsilon x_{2}^{2}+\epsilon^{2} x_{2}\right) w\left(x_{1}-2 \beta x_{2}^{2}, \frac{t^{\prime}}{2}-x_{2}\right), \\
w\left(y_{1}, y_{2}\right)= & \frac{1}{2 \sqrt{\pi y_{2}} \sqrt{\alpha_{1}+\alpha_{2} y+\alpha_{3} y^{2}}} \exp \left(\epsilon \int \frac{1}{\sqrt{\alpha_{1}+\alpha_{2} y+\alpha_{3} y^{2}}} d y\right) \\
& \times \exp \left(\frac{-\left(y_{1}-\int\left(d y / \sqrt{\alpha_{1}+\alpha_{2} y+\alpha_{3} y^{2}}\right)+\beta\left(t^{\prime}\right)^{2} / 2\right)^{2}}{4 y_{2}}\right) \\
& \times \exp \left\{-\frac{\beta(1-\beta)}{6}\left(t^{\prime}\right)^{3}+\frac{\beta \epsilon\left(t^{\prime}\right)^{2}}{2}+\frac{\epsilon^{2} t^{\prime}}{2}\right\} .
\end{aligned}
$$

Example 4.1. For $\beta=\epsilon=0$, the TDF for $\alpha_{3}>0$ and $\alpha_{1} / \alpha_{3}-\left(\alpha_{2} / 2 \alpha_{3}\right)^{2}>0$ can be simplified to

$$
\begin{aligned}
p\left(x, t ; y, t^{\prime}\right)= & \exp \left\{-\left(1 / 2 \alpha_{3}\left(t^{\prime}-t\right)\right)\left[\operatorname{arcsinh}\left(\left(\alpha_{2}+2 \alpha_{3} x\right) / \sqrt{4 \alpha_{1} \alpha_{3}-\alpha_{2}^{2}}\right)\right.\right. \\
& \times \frac{\left.\left.-\operatorname{arcsinh}\left(\left(\alpha_{2}+2 \alpha_{3} y\right) / \sqrt{4 \alpha_{1} \alpha_{3}-\alpha_{2}^{2}}\right)\right]^{2}\right\}}{\sqrt{2 \pi\left(t^{\prime}-t\right)} \sqrt{\alpha_{1}+\alpha_{2} y+\alpha_{3} y^{2}}} .
\end{aligned}
$$

Case 2. $k(x)=\beta x_{1}, \alpha(x)=\exp \left\{(\epsilon / 2)\left(\int\left(d x / \sqrt{\alpha_{1}+\alpha_{2} x+\alpha_{3} x^{2}}\right)\right)^{2}\right\}, \epsilon \neq 0$.

The corresponding stochastic differential equation for $x$ is

$$
\begin{aligned}
d x= & \left\{\left[\beta t+\epsilon \int \frac{d x}{\sqrt{\alpha_{1}+\alpha_{2} x+\alpha_{3} x^{2}}}\right] \sqrt{\alpha_{1}+\alpha_{2} x+\alpha_{3} x^{2}}+\frac{1}{4}\left(\alpha_{2}+2 \alpha_{3} x\right)\right\} d t \\
& +\sqrt{\alpha_{1}+\alpha_{2} x+\alpha_{3} x^{2}} d X .
\end{aligned}
$$


The solution to $(1.6 \mathrm{a})-(1.6 \mathrm{~b})$ is

$$
\begin{aligned}
p\left(x, t ; y^{\prime}, t^{\prime}\right)= & \exp \left\{-\frac{\epsilon}{2}\left(\int \frac{d x}{\sqrt{\alpha_{1}+\alpha_{2} x+\alpha_{3} x^{2}}}\right)^{2}\right\} \exp \left\{-\beta t \int \frac{d x}{\sqrt{\alpha_{1}+\alpha_{2} x+\alpha_{3} x^{2}}}\right\} \\
& \times z\left(\int \frac{d x}{\sqrt{\alpha_{1}+\alpha_{2} x+\alpha_{3} x^{2}}}, \frac{t}{2}\right),
\end{aligned}
$$

where

$$
\begin{aligned}
z\left(x_{1}, x_{2}\right)= & \exp \left(\frac{\epsilon}{2} x_{1}^{2}+2 \beta x_{1} x_{2}+\frac{4}{3} \beta(1-\beta) x_{2}^{3}\right) \\
& \times w\left(\left(x_{1}+\frac{2 \beta}{\epsilon} x_{2}+\frac{\beta}{\epsilon^{2}}\right) e^{-2 \epsilon x_{2}}, \frac{1}{4 \epsilon}\left(e^{-4 \epsilon x_{2}}-e^{-2 \epsilon t^{\prime}}\right)\right), \\
w\left(y_{1}, y_{2}\right)= & \frac{\exp \left(-\beta(1-\beta)\left(\left(t^{\prime}\right)^{3} / 6\right)-\epsilon t^{\prime}\right)}{2 \sqrt{\pi y_{2}} \sqrt{\alpha_{1}+\alpha_{2} y+\alpha_{3} y^{2}}} \\
& \times \exp \left\{-\frac{\left(y_{1}-\left[\int\left(d y / \sqrt{\alpha_{1}+\alpha_{2} y+\alpha_{3} y^{2}}\right)+\beta t^{\prime} / \epsilon+\beta / \epsilon^{2}\right] e^{-\epsilon t^{\prime}}\right)^{2}}{4 y_{2}}\right\} .
\end{aligned}
$$

\section{Summary of results}

In this section, we summarize the results of this paper by quoting the equation numbers for the solutions $p\left(x, t ; y, t^{\prime}\right)$ to (1.6a)-(1.6b) for given functions $A(x, t)$ and $B(x, t)$. These solutions can be viewed as TDFs for processes $x$ that follow (1.4).

5.1. $B(x)=c x^{\gamma}, \gamma \neq 1$.

5.1.1. $A=A(x)$. The solutions found for $A=A(x)$ depend on the form of $A^{\prime}-(2 \gamma / x) A+$ $\left(1 / c^{2} x^{2 \gamma}\right) A^{2}$.

\begin{tabular}{l|c}
\hline$A^{\prime}-\frac{2 \gamma}{x} A+\frac{1}{c^{2} x^{2 \gamma}} A^{2}$ & $\begin{array}{c}\text { Equation number for } \\
p\left(x, t ; y, t^{\prime}\right)\end{array}$ \\
\hline$m x^{2 \gamma-2}+n$ & \\
$\alpha x^{2 \gamma-2}+\beta x^{2-2 \gamma}+\epsilon$ where $\beta>0$ & \\
$\alpha x^{2 \gamma-2}+\beta x^{2-2 \gamma}+\epsilon$ where $\beta<0$ & \\
$\alpha x^{2-2 \gamma}+\beta x^{1-\gamma}+\epsilon+m x^{2 \gamma-2}$ where $\alpha=0$ & \\
$\alpha x^{2-2 \gamma}+\beta x^{1-\gamma}+\epsilon+m x^{2 \gamma-2}$ where $\alpha>0$ & \\
$\alpha x^{2-2 \gamma}+\beta x^{1-\gamma}+\epsilon+m x^{2 \gamma-2}$ where $\alpha<0$ &
\end{tabular}


5.1.2. $A=A(x, t)$.

\begin{tabular}{l|c}
\hline$A(x, t)$ & $\begin{array}{c}\text { Equation number for } \\
p\left(x, t ; y, t^{\prime}\right)\end{array}$ \\
\hline$c^{2}\left(\frac{\gamma}{2} x^{2 \gamma-1}+\epsilon x^{\gamma}+\beta(1-\gamma) x^{\gamma} t\right)$ & \\
$c^{2}\left(\frac{\gamma}{2} x^{2 \gamma-1}+\epsilon x+\beta(1-\gamma) x^{\gamma} t\right), \epsilon \neq 0$ \\
$c^{2}\left(\frac{\gamma}{2} x^{2 \gamma-1}+\epsilon x^{\gamma}+\beta(2-2 \gamma) x t\right), \beta>0$ \\
$c^{2}\left(\frac{\gamma}{2} x^{2 \gamma-1}+\epsilon x+\beta(2-2 \gamma) x t\right), \beta>0$ \\
$c^{2}\left(x^{2 \gamma-1}\left(1-\frac{\gamma}{2}\right)+\beta(2-2 \gamma) x t\right), \beta>0$
\end{tabular}

5.2. $B(x)=\sqrt{\alpha_{1}+\alpha_{2} x+\alpha_{3} x^{2}}$.

\begin{tabular}{l|l}
\hline$A(x, t)$ & $\begin{array}{l}\text { Equation number for } \\
p\left(x, t ; y, t^{\prime}\right)\end{array}$ \\
\hline$(\beta t+\epsilon) \sqrt{\alpha_{1}+\alpha_{2} x+\alpha_{3} x^{2}}+\frac{1}{4}\left(\alpha_{2}+2 \alpha_{3} x\right)$ & \\
$\left(\beta t+\epsilon \int \frac{d x}{\sqrt{\alpha_{1}+\alpha_{2} x+\alpha_{3} x^{2}}}\right) \sqrt{\alpha_{1}+\alpha_{2} x+\alpha_{3} x^{2}}$ & \\
$+\frac{1}{4}\left(\alpha_{2}+2 \alpha_{3} x\right), \quad \epsilon \neq 0$ & \\
\hline
\end{tabular}

\section{Concluding remarks}

In this paper we have demonstrated how it is possible to find fundamental solutions to the backward Kolmogorov equations by reducing the equations to their canonical form. In all our examples, (1.6c) is satisfied and so these fundamental solutions are interpretable as transition density functions (TDFs) for variables following certain stochastic processes.

In this way, we were able to extend the list of fundamental solutions and TDFs for the processes (1.4) considered by Craddock and Platen, that is, $B=c x^{1 / 2}$, and where $A=f(x)$ satisfies (1.13a)-(1.13c), and also find fundamental solutions and TDFs for the more general cases when $B=c x^{\gamma}$, and $B=\sqrt{\alpha_{1}+\alpha_{2} x+\alpha_{3} x^{2}}$, and when $A$ is time-dependent. Some of these more general cases have proven to agree well with real financial data.

\section{References}

[1] C. Albanese, G. Campolieti, P. Carr, and A. Lipton, Black-Scholes goes hypergeometric, Risk 14 (2001), no. 12, 99-103.

[2] C. Albanese and A. Kuznetsov, Transformations of Markov processes and classification scheme for solvable driftless diffusions, preprint, http://www3.imperial.ac.uk/mathfin/people/calban/ papersmathfin. 


\section{Fundamental solutions to Kolmogorov equations}

[3] S. Beckers, The constant elasticity of variance model and its implications for option pricing, Journal of Finance 35 (1980), no. 3, 661-673.

[4] F. Black and M. J. Scholes, The pricing of options and corporate liabilities, Journal of Political Economy 81 (1973), no. 3, 637-654.

[5] G. W. Bluman, On the transformation of diffusion processes into the Wiener process, SIAM Journal on Applied Mathematics 39 (1980), no. 2, 238-247.

[6] K. C. Chan, A. Karolyi, F. Longstaff, and A. B. Sanders, An empirical comparison of alternative models of the short-term interest rate, Journal of Finance 47 (1992), no. 3, 1209-1227.

[7] J. C. Cox, J. E. Ingersoll Jr., and S. A. Ross, A theory of the term structure of interest rates, Econometrica 53 (1985), no. 2, 385-407.

[8] J. C. Cox and S. A. Ross, The valuation of options for alternative stochastic processes, Journal of Financial Economics 3 (1976), no. 1-2, 145-166.

[9] M. Craddock and E. Platen, Symmetry group methods for fundamental solutions, Journal of Differential Equations 207 (2004), no. 2, 285-302.

[10] J. Fan, J. Jiang, C. Zhang, and Z. Zhou, Time-dependent diffusion models for term structure dynamics, Statistica Sinica 13 (2003), no. 4, 965-992.

[11] W. Feller, Diffusion processes in genetics, Proceedings of the Second Berkeley Symposium on Mathematical Statistics and Probability, 1950 (J. Neyman, ed.), University of California Press, California, 1951, pp. 227-246.

[12] _ Two singular diffusion problems, Annals of Mathematics. Second Series 54 (1951), no. 1, $173-182$.

[13] J. Goard and N. Hansen, Comparison of the performance of a time-dependent short-interest rate model with time-independent models, Applied Mathematical Finance 11 (2004), 147-164.

[14] T. Ho and S. Lee, Term structure movements and pricing interest rate contingent claims, Journal of Finance 41 (1986), no. 5, 1011-1029.

[15] N. H. Ibragimov, A. V. Aksenov, V. A. Baikov, V. A. Chugunov, R. K. Gazizov, and A. G. Meshkov, CRC Handbook of Lie Group Analysis of Differential Equations. Vol. 2, edited by N. H. Ibragimov, CRC Press, Florida, 1995.

[16] P. Wilmott, Derivatives: The Theory and Practice of Financial Engineering, John Wiley \& Sons, West Sussex, 1998.

Joanna Goard: School of Mathematics and Applied Statistics, University of Wollongong, Wollongong, NSW 2522, Australia

E-mail address: joanna@uow.edu.au 


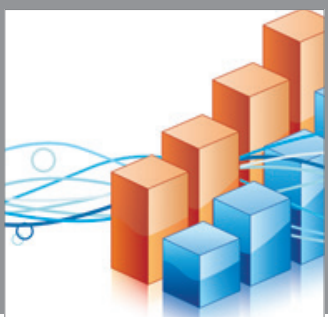

Advances in

Operations Research

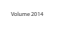

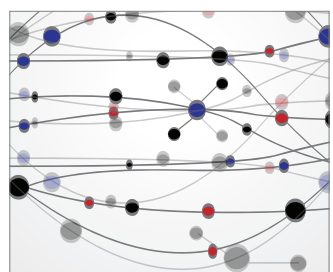

\section{The Scientific} World Journal
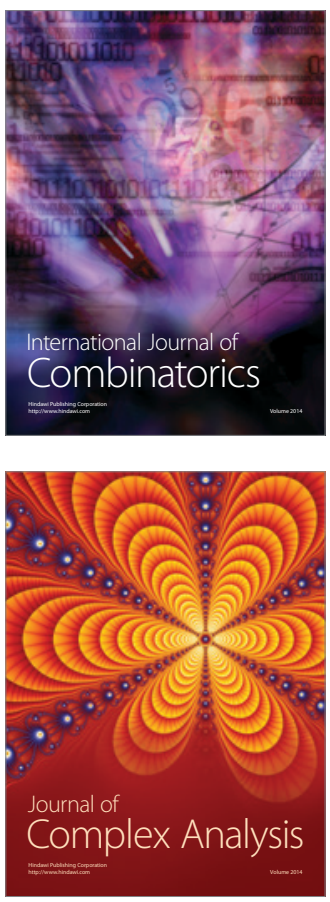

International Journal of

Mathematics and

Mathematical

Sciences
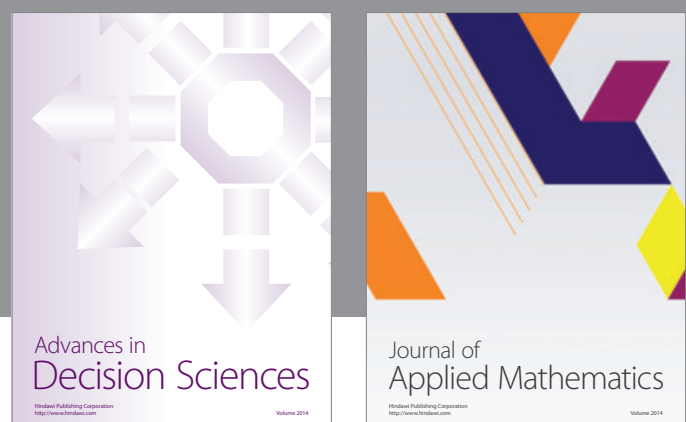

Journal of

Applied Mathematics
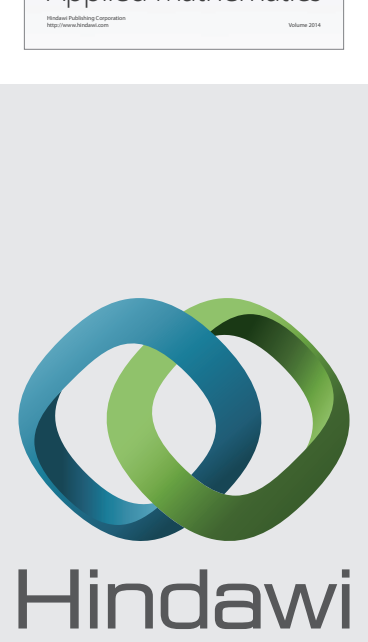

Submit your manuscripts at http://www.hindawi.com
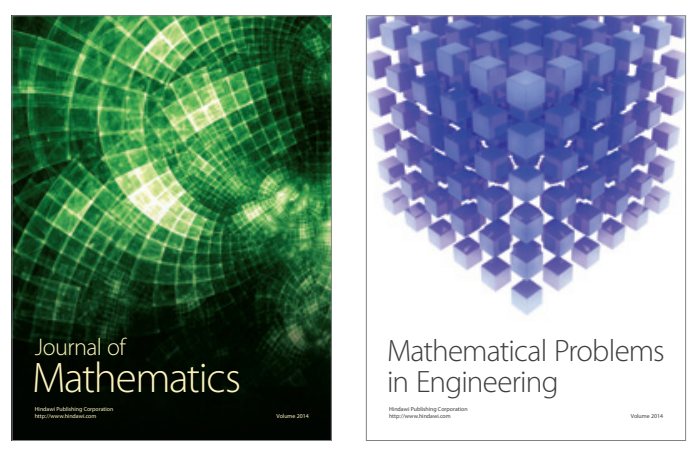

Mathematical Problems in Engineering
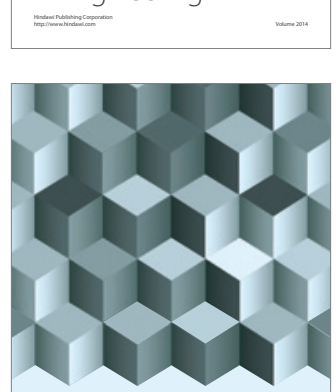

Journal of

Function Spaces
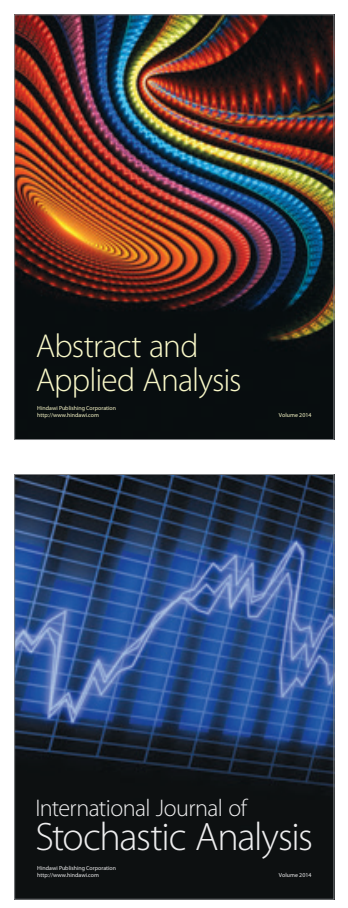

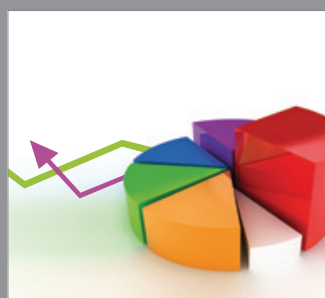

ournal of

Probability and Statistics

Promensencen
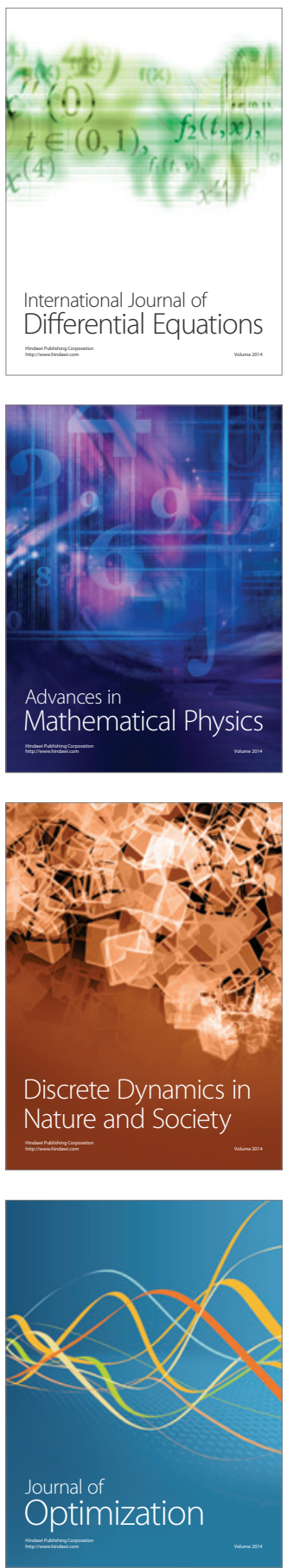